\title{
Positives from Online Teaching
}

\author{
The rapid switch to online teaching earlier this year was a huge and \\ stressful adjustment, but physics teachers also learned valuable new tools \\ and practices.
}

W

hen the pandemic forced schools, colleges, and universities to switch to remote learning, many teachers had to rapidly reinvent their lesson plans. Lab classes or anything hands-on and group oriented were particularly tricky to pull off. But teachers and professors were inventive. And in some cases, they discovered new assignments or ways of communication that worked so well they were worth keeping even if and when in-person classes resume. As students head back to school-online, in-person, or somewhere in between - we asked educators to share some of the positive lessons they learned.

\section{- Jessica Thomas, Editor}

\section{Group Dynamics Matter}

My team develops introductory labs for undergraduates. In the shift to remote instruction, I found that the biggest loss was in the interactions with and between students. As we prepare for continued online instruction in the Fall, we are therefore deliberately structuring group interactions.

For example, we used to randomly assign students to groups of two to three. We'd then have students change groups a couple of times each semester. This year, we'll instead survey students about their group-work preferences, asking whether they prefer to take a particular role, have a team leader, tinker with equipment before deciding what to do, and so on. These responses will help us form the groups. A couple of weeks into the semester, students will be asked to evaluate their group-work experiences, themselves, and their team. We will intervene if there are any issues. Each group will document how its members are participating in lab activities, so that they can consider whether their group work is fair and equitable. Finally, we will create ways for the students to check in with other groups and discuss their work.
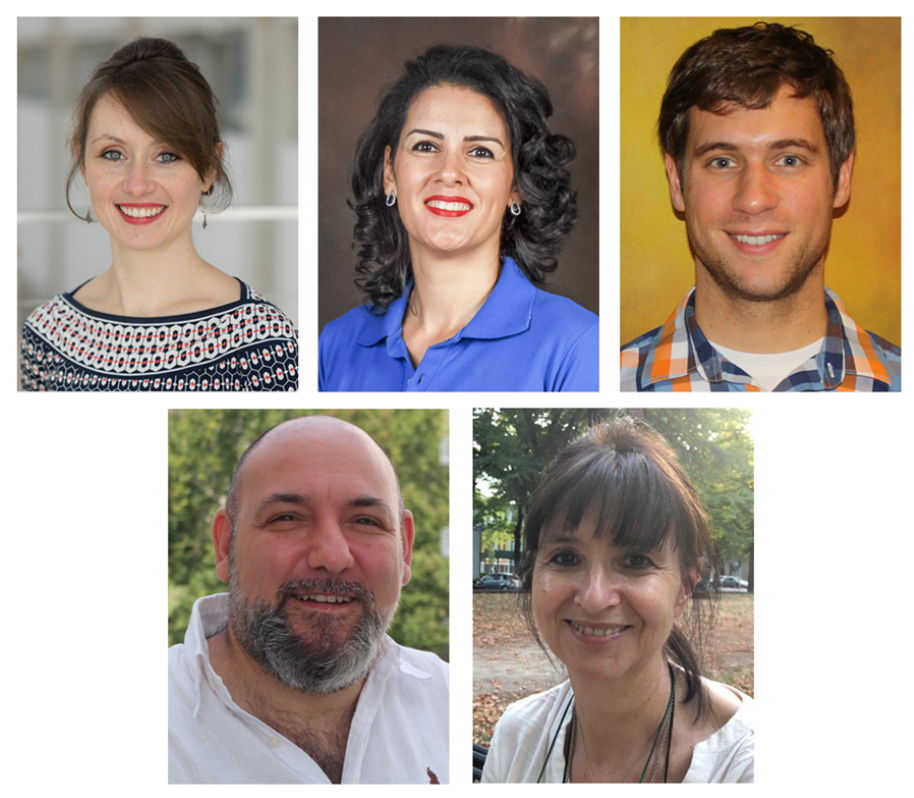

Clockwise from top left: Natasha Holmes, Andrea de Camargo, Ryan Terrien, Fabrizio Villa, and Laila Evangelisti.

Credit: C. Kitchen, D. Marques, Carlton College, F. Villa, L. Evangelisti

These structures would have seemed burdensome before the pandemic. Now, they seem necessary to support students' sense of community and sense of belonging. It's also become clear that this kind of structure is important no matter how we teach, so we'll continue to use it when we return to in-person instruction.

- Natasha Holmes is the Ann S. Bowers Assistant Professor of Physics at Cornell University.

New Tools for On-the-road Teaching Last Spring, I taught two physics courses for undergraduate 
chemistry students, one covered theory, the other was a lab course on applied electromagnetism and optics. The theory course went reasonably well when we had to switch to online teaching. I am less satisfied with the outcome for the lab. This four-month course is quite demanding: students are supposed to complete eight "practices"-a collection of experiments performed over four hours. We were only on the second practice when the pandemic hit.

The institute's media team helped us professors record demonstrations of some of the experiments. We then sent these videos along with the "results" that we collected to the students, asking them to discuss what they saw and answer questions. Later, I had to resort to slides and demonstrations that were already available on the internet.

We're planning to do a compact version of the in-person labs early next year, covering less material and with me doing lab demonstrations that students watch. It's far from ideal. Still, the positive in this whole experience is that we had to learn new communication and teaching tools that we can use later. Previously, when I travelled, I had to find a substitute teacher for my class. Now I know that I can teach the class virtually while I'm on the road. Plus, my students were really appreciative of my online-teaching efforts: I got a surprising number of "Thank you for your dedication" notes.

\section{- Andrea de Camargo is a Professor of Physics at the São Carlos} Institute of Physics, University of São Paulo, Brazil.

\section{The Creative Freedom of Blog Posts}

Our advanced laboratory course for undergrads emphasizes written, oral, and visual communication. During normal years, students both presented papers from the experimental physics literature and shared their final projects in a poster session. But I couldn't rely on either of these in-person formats last Spring. Instead, I replaced them with an assignment to write blog posts about papers and about the final projects. The students then shared their posts with the class.

This format has a few attractive features. It requires "comments" from classmates, which means the writers are accountable to their peers. It also works for students regardless of the time zone they are in or the bandwidth they have access to. And it's an opportunity for students to practice written and

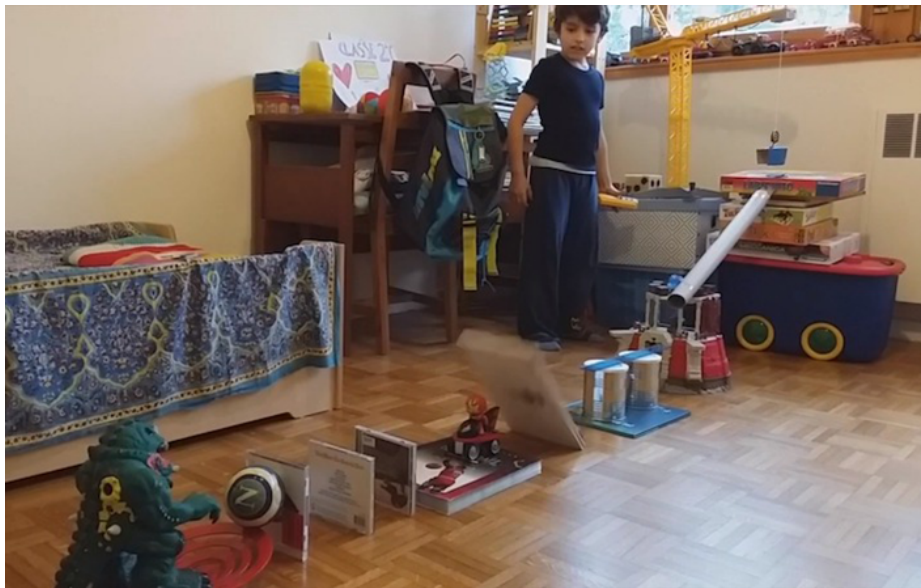

A young student tests a home-built "chain reaction." Credit: INAF

visual communication. Importantly, this type of writing differs from formal lab writeups in that students knew they were writing directly for their classmates.

Feedback from students after the class indicated that many enjoyed the chance to express their ideas more creatively than formal scientific writing typically allows. Another potential benefit of this format is the ease with which it can be integrated into an electronic portfolio (an online collection of student work). For these reasons, I am excited to continue using blog posts even when I'm back to teaching this class in person.

\section{- Ryan Terrien is an Assistant Professor of Physics and Astronomy at Carleton College in Minnesota.}

\section{Tinkering Goes Online}

Starting in 2014, the Italian National Institute of Astrophysics (INAF) partnered with primary schools in Bologna on a creative approach to STEM education. Known as tinkering, the initiative helps young children explore science in a playful, immersive way. In their classrooms, 6-to-12-year-old students build devices and solve science problems without following a pre-determined recipe. They are guided by their teachers and by local astrophysicists who collaborate with ALMA-the largest radio telescope in the world.

Science quickly became one of the favorite subjects for the kids 
in the tinkering program. But when the lockdown started, we weren't sure we could successfully bring the activities online because they rely so heavily on in-person interactions among the students and with the facilitators. Also, kids at home don't necessarily have access to the same resources and support that public schools provide. But we forged ahead with a "chain reaction" project. We asked students to prepare short videos of the domino-like falling of nearby objects-illustrating the cause-and-effect relationship that is the cornerstone of the scientific method.

We were thrilled with the outcome. The participation of most children was active and meaningful. We also developed videos and training resources that could help us make tinkering more effective and broadly available. Italy now plans to reopen schools, and we hope to resume the activities in the classroom. But we will develop the online materials as well. These resources could guide teachers in the most remote regions of Italy, spurring the creativity of more of our littlest explorers of the Universe.

- Laila Evangelisti is a teacher at the IC12 public school of Bologna, Italy, and Fabrizio Villa is a Senior Researcher at the Italian National Institute for Astrophysics (INAF) of the Astrophysics and Space Science Observatory of Bologna. 